\title{
Características zootécnicas e potenciais do tambaqui (Colossoma macropomum) para a piscicultura brasileira
}

\author{
${\text { Bruna Barçante }{ }^{1 *} \text { \& Alexandre Benvindo de Sousa }}^{2}$ \\ ${ }^{1}$ Graduanda em Aquacultura, Escola de Veterinária, UFMG. \\ ${ }^{2}$ Professor Associado do COLTEC, UFMG. \\ *Autor para correspondencia, E-mail: brunabarcante@vet.grad.ufmg.br
}

\begin{abstract}
RESUMO: A aquacultura no Brasil vem crescendo em ritmo acelerado, devido às condições naturais favoráveis que o país apresenta para o desenvolvimento da atividade. Grande parte da produção aquícola brasileira é representada pela aquacultura continental, principalmente pela piscicultura, que ainda é amplamente dominada por espécies exóticas. O Brasil possui diversas espécies nativas com grande potencial para a produção em cativeiro e, dentre elas, está o tambaqui, Colossoma macropomum, que é a espécie nativa mais produzida no país e que vem ocupando papel de destaque para a piscicultura não só no Brasil, como em outros países. O tambaqui é nativo das bacias dos rios Amazonas e Orinoco e é bastante apreciado devido ao seu rápido crescimento, adaptação ao cativeiro, fácil treinamento alimentar, resistência, além do alto valor comercial e gastronômico de sua carne. Hoje a sua produção representa $14 \%$ do total de pescado proveniente da piscicultura continental brasileira e diversos recursos tecnológicos já vêm sendo utilizados para a otimização técnica e econômica da sua produção.
\end{abstract}

Palavras-chave: Tambaqui, potencial zootécnico, aquacultura.

\section{Zootechnical Characteristics and potential of tambaqui (Colossomamacropomum) to Brazilian fish culture}

\begin{abstract}
Aquaculture has been growing fast in Brazil due propitious nature conditions presented to such economic activity. Most of Brazilian production is due to continental aquaculture, especially the fish culture, and is widely focused on exotics species. There are many native species with high breeding potential among them, the tambaqui, Colossoma macropomum, which is the most native species cultivated and has been taking a relevant role in Brazil and in others countries, as well. Tambaqui is native from Amazonas and Orinoco river basins and very appreciated because its fast development, captive fitness, easy training food, resistant, and its high commercial and gastronomy meat value. Nowadays its production represents $14 \%$ of Brazilian continental fish culture and several technologies resources has been used to optimize technically and economically its production.
\end{abstract}

Key words: Tambaqui, Zoothecnical potential, aquaculture.

\section{Introdução}

A demanda por pescado tem crescido significativamente nos últimos e isso se deve à mudança dos hábitos alimentares da população, quem vem buscando alimentos saudáveis para incluir em sua dieta, já que a carne de pescado é rica em proteínas e ômega-3. Nesse sentido, a aquacultura surge como a atividade zootécnica capaz de contribuir para a segurança alimentar no planeta. Além disso, é considerada atividade econômica adequada para pequenos, médios e grandes produtores, uma vez que a produtividade em relação à área ocupada é superior àquela que se verifica em outras criações como bovinos, aves, suínos, caprinos etc. No Brasil tem se verificado o desenvolvimento da atividade aquícola. Esse desenvolvimento é favorecido pelas condições naturais que o país apresenta pela grande disponibilidade hídrica e seus diversos microclimas. Cabe ainda ressaltar que a pesca extrativista brasileira, assim como no resto do mundo, está estagnada e possui limitadas condições de expansão devido ao risco de 
esgotamento dos recursos pesqueiros e populações nativas.

A aquacultura continental é a mais representativa no Brasil, com maior destaque para a piscicultura. $O$ país possui diversas espécies de peixes nativos com grande potencial para a produção em cativeiro. Porém, há muitas espécies sendo produzidas sem estudos que as coloque em plena viabilidade zootécnica para a sua produção, por isso a aquacultura brasileira ainda é dominada por espécies exóticas. O tambaqui (Colossoma macropomum) e o pacu (Piaractus mesopotamicus) são espécies nativas que vêm recebendo grande atenção devido as suas características favoráveis para a produção em cativeiro. Também merecem relato os híbridos dessas espécies, paqui e tambacu. Por sua vez, o tambaquié a espécie nativa que recebe maior destaque na piscicultura brasileira sendo aquela mais produzida no país.

Nesta revisão abordamos o potencial e as características zootécnicas do tambaqui para a produção em cativeiro e as razões, pela qual, essa espécie ocupa papel relevante na piscicultura brasileira.

\section{Aspectos biológicos do tambaqui}

As espécies nativas da região amazônica são de grande difusão não só no Brasil, mas pelo mundo devido ao seu porte, rápido crescimento, alta resistência e sabor da sua carne. (Mendonça et al., 2009). O tambaqui, Colossoma macropomum, peixe tropical nativo das bacias dos rios Amazonas e Orinoco pertence à classe Osteichthyes, ordem Characiformes e família Serrasalmidae. É o segundo maior peixe de escamas de água doce da América do Sul e vem sendo amplamente difundido em diversas regiões do país e do continente sul-americano devido ao crescimento e desenvolvimento da piscicultura (Dairiki et al., 2011). As águas claras das bacias dos rios Amazonas e Orinoco são seu hábitat favorito, mas podem migrar para as águas escuras para se alimentarem. (Casanova, 2008). O $C$. macropomum possui hábitos onívoros, se alimentando preferencialmente de frutos e sementes, sendo, por isso, considerado "símbolo ictíco da floresta tropical”. (Dairiki et al., 2011). $\mathrm{O}$ tambaqui desenvolveu dentes molariformes, adaptados para quebrar sementes duras e numerosas cerdas branquiais, utilizadas para a retenção de zooplâncton, dos quais se alimenta. Em geral, os juvenis de tambaqui são onívoros e se alimentam de frutas, sementes e organismos do zooplâncton, mas também podem filtrar o fitoplâncton. Quando adultos, alimentam-se exclusivamente de sementes, o que faz do tambaqui um importante dispersor de sementes. Eles aceitam facilmente alimentos artificiais em cativeiro, além de possuírem uma boa reprodutividade e apresentarem cuidado parental. (Gomes et al., 2006).

O tambaqui possui uma alta popularidade, o que pode ser atribuída às suas características zootécnicas favoráveis para a aquacultura, a saber: a facilidade de produção de alevinos, seu rápido crescimento em cativeiro, rusticidade, resistência a elevadas temperaturas na água dos sistemas de cultivo e a eventuais quedas de oxigênio dissolvido na água, ao manuseio, às enfermidades e facilidade de comercialização, além do elevado valor de sua carne (Paula, 2009; Mendonça et al., 2009). Em situações de hipóxia, baixo teor de oxigênio dissolvido na água, o tambaqui apresenta uma adaptação morfológica, o aumento do lábio inferior, que garante sua sobrevivência e também costuma nadar próximo à superfície para captar mais oxigênio, além de reduzir seu metabolismo e sua taxa de crescimento. (Dairiki et al., 2011).

\section{Aspectos de manejo e cultivo do tambaqui}

O tambaqui pode ser criado em diferentes sistemas de cultivo, sendo alguns deles a produção intensiva em viveiros escavados, barragens e tanques-rede, sendo a utilização de rações balanceadas de acordo com as características da espécie essencial para o sucesso da produção. (Dairiki et al., 2011). Os filés de tambaquis, criados sob altas taxas de renovação de água, apresentam menos gordura do que aqueles criados em sistemas sem renovação de água, e maior acúmulo de proteína corporal. (Paula, 2009).

"Viveiros semiescavados são sistemas viáveis para a produção de tambaqui pela não agressão às áreas de preservação permanentes (APP's) por exigirem apenas reservatório de água para abastecimento (Resolução CONAMA 369/06) e pela quantidade de efluente gerado ser mínima, pois os viveiros demandam de água apenas para reposição e sua renovação total só ocorre após quatro ciclos de produção. Pode haver o reuso da água e o sistema otimiza o monitoramento da qualidade da água e emprego das boas práticas de manejo (Cavero et al.2009)". 
A produção de tambaqui é dividida, de forma geral, em larvicultura, alevinagem e engorda. A larvicultura compreende a fase em que os peixes são criados da eclosão até o peso médio individual (PMI) de 0,5 a $1 \mathrm{~g}$ durante 30 a 45 dias. A alevinagem, ou produção de juvenis, dura cerca de 60 dias e o PMI dos peixes fica entre 40 g e 50 g. Na engorda tempo é variável, dependendo do peso de abate. O ciclo de produção do tambaqui pode ser concluído de $10 \mathrm{a}$ 12 meses, com densidade de estocagem de 10 juvenis $/ \mathrm{m}^{2}$, taxa média de sobrevivência de $85 \%$, densidade de engorda de 3.250- 4000 peixes/ha, peso médio de venda de $1,8 \mathrm{~kg}$ em 10 meses de engorda e $3,1 \mathrm{~kg}$ em 12 meses, com rendimento de $10.075 \mathrm{~kg} / \mathrm{ha} / \mathrm{ano}$ (Dairiki et al., 2011; Melo et al., 2001).

Sua produção despertou o interesse empresarial devido à boa rentabilidade, pois, pode alcançar Taxa Interna de Retorno (TIR) acima de $40 \%$ por safra de comercialização e Período de Recuperação do Capital (PRC) abaixo de três anos. (Izel et al., 2004). Diversas estratégias vêm sendo desenvolvidas com o objetivo de aumentar a produtividade e rentabilidade do cultivo de tambaqui, com o intuito de coloca-lo em um patamar de plena viabilidade zootécnica para a piscicultura brasileira. Recursos tecnológicos vêm sendo utilizados na produção da espécie já demonstrando capacidade de incremente na produtividade e diminuição dos custos da produção como, por exemplo, a adoção de sistema de aeradores nos sistemas de cultivo, utilização de enzimas exógenas e dimensionamento de viveiros. (Cavero et al., 2009).

O tambaqui, pode ser criado em tanques-rede com volumes de 1 e $6 \mathrm{~m}^{3}$, apresentando boa adaptação (Gomes et al., 2004) e, para a sua recria em tanques-rede, a densidade de 400 peixes $/ \mathrm{m}^{3}$ é a mais adequada (Brandão et al., 2004) sendo recomendável oferecer $10 \%$ da biomassa, durante o manejo alimentar, dividida em três refeições diárias. Na fase de engorda em tanques-rede, o tambaqui pode atingir $950 \mathrm{~g}$ em 240 dias de criação, com conversão alimentar de 1,8 e produção de $46,8 \mathrm{~kg} / \mathrm{ha}$ (Gomes et al., 2006). Embora se tenha conhecimento da densidade de estocagem adequada para a criação de tambaquis em tanques-rede, na fase de recria e do volume do tanque-rede para obtenção de boa produtividade, ainda faltam informações precisas sobre o manejo alimentar adequado para evitar o desperdício de ração e para maximizar a produção nesse sistema de cultivo. Certamente esse fator deverá ser dependente de aspectos de manejo, variáveis de qualidade de água e da qualidade da ração. Estudos realizados em tanques-rede em lagos de várzea da Amazônia Central demonstraram que o fornecimento de ração na taxa de $5 \%$ do peso vivo/dia durante a fase inicial de engorda é satisfatório para o desenvolvimento dos juvenis (Chagas et al., 2005).

Observa-se no Brasil o crescimento contínuo da produção de tambaqui, partindo de 8 mil toneladas em 1994 e atingindo 46 mil toneladas em 2009. A produção da espécie cresceu $123 \%$ entre 2003-2009, sendo que hoje a produção de tambaqui representa $14 \%$ do total de pescado proveniente da piscicultura continental. (Dairiki et al., 2011).

\section{Considerações finais}

Embora a piscicultura brasileira seja largamente realizada com espécies exóticas que respondem a maior parte da produção pesqueira no país, existem espécies nativas com que se destacam para a produção em cativeiro, dentre elas o tambaqui, que teve um incremento de sua produção maior que $40 \%$ entre os anos de 2007 2009. O tambaqui, dentre as espécies nativas brasileiras, é a que mais vem recebendo destaque na piscicultura brasileira e é bastante apreciada também entre os países Latinos Americanos. As características biológicas da espécie, associadas as zootécnicas (prolificidade, rusticidade, adaptabilidade ao cativeiro, boa qualidade de carne, alto valor comercial) fazem do tambaqui uma espécie nativa de grande importância para a piscicultura brasileira, com potencial para aumentar sua participação na produção de pescado nacional, visando atender os mercados interno e externo.

\section{Referências bibliográficas}

Brandão, F.R., Gomes, L.C., Chagas, E.C. \& Araújo, L.D. 2004. Densidade de estocagem de juvenis de tambaqui durante a recria em tanques-rede. Pesquisa Agropecuária Brasileira, 39, 357-362.

Casanova, F.M. Caracterização in silico de biossensores em Colossoma macropomum: diagnóstico molecular e monitoramento de ambientes impactados. Dissertação (Parte dos requisitos para obtenção do título de mestre 
em Ciências Biológicas, área de concentração em Genética, Conservação e Biologia Evolutiva). Ministério da ciência e tecnologia. Instituto nacional de pesquisas da Amazônia. Manaus, AM. 2008.

Cavero, B.A., Rubim, M.A.L \& Pereira, T.M. 2009. Criação comercial do tambaqui Colossoma macropomum (Cuvier, 1818). Manejo e sanidade de peixes em cultivo. Marcos Tavares-Dias/Organizador. Macapá. Empresa Brasileira de Pesquisa AgropecuáriaEMBRAPA/Amapá.

Chagas, E.C., Gomes, L.C., Martins Júnior, H., Roubach, R. \& Lourenço, J.N.P. 2005. Desempenho de tambaqui cultivado em tanques-rede, em lago de várzea, sob diferentes taxas de alimentação. Pesquisa Agropecuária Brasileira, 40, 833-835.

Dairiki, J. \& Silva, T,B.A.. Revisão de literatura: exigências nutricionais do tambaqui compilação de trabalhos, formulação de ração adequada e desafios futuros. - Manaus: Empresa Brasileira de Pesquisa AgropecuáriaEMBRAPA Amazônia Ocidental, 2011.44 p. - (Embrapa Amazônia Ocidental. Documentos; 91).

Gomes, L. C., Brandão, F. R., Chagas, E. C., Ferreira, M. F. B. \& Lourenço, J. N. P. 2004. Efeito do volume do tanque-rede na produtividade de tambaqui (Colossoma macropomum) durante a recria. Acta Amazônica, 34, 111-113.

Gomes, L.C., Araujo-Lima, C.A.R. M., ChippariGomes, A.R. \& Roubach, R. 2006. Transportation of juvenile tambaqui (Colossomamacropomum) in a closed system. Brazilian Journal of Biologie, 66, 493-502.

Izel, A.C.U. \& Melo, L.A.S. 2004. Criação de tambaqui (Colossoma macropomum) em tanques escavados no estado do Amazonas.
Manaus: Embrapa Amazônia Ocidental, 19p. (Embrapa Amazônia Ocidental. Documentos, 32).

Melo, L.A.S., Izel, A.C.U. \& Rodrigues, F.M. 2001. Criação de tambaqui (Colossoma macropoтum) em viveiros de argila/barragens no estado do Amazonas. Manaus: Empresa Brasileira de Pesquisa AgropecuáriaEMBRAPA Amazônia Ocidental, 30 p.

Mendonça, P.P., Ferreira, R.A., Junior, M.V. Vidal; Andrade, D.R., Santos, M.V.D., Ferreira, A.V. \& Rezende, F.P. 2009. Influência do fotoperíodo no desenvolvimento de juvenis de tambaqui (Colossoma macropomum). Archivos de Zootecnia, 58, 323-331.

Paula, F.G. Desempenho do tambaqui (Colossoma macropomum), da pirapitinga (Piaractus brachypomum) e do híbrido tambatinga (C. macropomum $\mathrm{x} P$. brachypomum) mantidos em viveiros fertilizados na fase de engorda. Dissertação (requisito parcial para obtenção do título Mestre em Ciência Animal). Universidade Federal de Goiás, Escola de Veterinária, 2009. Bibliografia: f. 50-57.

\section{Recebido em Março 20, 2014}

Aceito em Novembro 4, 2014

License information: This is an open-access article distributed under the terms of the Creative Commons Attribution License, which permits unrestricted use, distribution, and reproduction in any medium, provided the original work is properly cited 